\title{
Osteoma osteoide, causa inusual de dolor de muñeca
}

\section{Osteoid osteoma, Unusual Cause of Wrist Pain}

\author{
Teresa María Beteta Robles ${ }^{1}$ Angel García Olea ${ }^{2}$ Luz Maria Moran Blanco ${ }^{3}$
}

${ }^{1}$ Servicio Traumatología, Hospital Universitario Puerta de Hierro

Majdahonda, Madrid, España

Address for correspondence Teresa María Beteta Robles, Servicio Traumatología, Hospital Universitario Puerta de Hierro Majdahonda,

2 Unidad de cirugía de la mano, Traumatología, Hospital Univeritario Madrid, España, Spain (e-mail: teresa.beteta@gmail.com).

Puerta de Hierro Majadahonda, Madrid, España

${ }^{3}$ Unidad radiología musculoesquelética, Hospital Univeritario Puerta de Hierro Majadahonda, Madrid, España

Rev Iberam Cir Mano 2018;46:46-51.

\author{
Resumen \\ Palabras Clave \\ - osteoma osteoide \\ - dolor \\ - muñeca
}

Presentamos el caso de una mujer joven con dolor en muñeca de meses de evolución. Las pruebas de imagen que se le realizan no son concluyentes. La clínica nos hace sospechar en el $\mathrm{OO}$ como posible diagnostico por lo que se realiza una TC que pone de manifiesto la presencia del nidus. Siguiendo el protocolo de nuestro servicio, se decide como tratamiento la ablación percutánea por radiofrecuencia. La mejoría clínica es inmediata. Aunque la mayoría de los $\mathrm{OO}$ son de diagnóstico sencillo, siguen presentándose algunos casos, como el del paciente expuesto, cuyas localizaciones peculiares o tamaño pequeño pueden producir errores o retrasos en el diagnostico.

We present the case of a young woman with pain in the wrist of months of evolution. The imaging studies did not show pathology. The symptoms makes us suspect in the $\mathrm{OO}$ as possible diagnosis. ACT is made, and shows The presence of the nidus. Following the protocol of our service, percutaneous radiofrequency ablation is decided as a treatment. Clinical improvement is immediate. Although most of the OOs are easy to diagnose, there are still some cases, such as the exposed patient, whose peculiar place or small size can cause errors or delays in the diagnosis.

\section{Introducción}

El osteoma osteoide $(\mathrm{OO})$ es un tumor óseo benigno de pequeño tamaño, entre y $2 \mathrm{~cm}$. Se caracteriza por un nidus bien vascularizado compuesto por tejido conectivo y trabéculas entrelazadas de osteoide y hueso calcificado rodeado de osteoblastos. Tienen un potencial de crecimiento limitado y un dolor desproporcionado. Suponen aproximadamente el 11\% de los tumores benignos y el 3\% de todos los tumores óseos primarios. $^{1,2}$

Es más común en el sexo masculino y el rango de edad, aunque es amplio, es infrecuente en menores de 5 años y en mayores de 30 años. Se puede afirmar que la edad más susceptible es de 10 a 25 años. ${ }^{1}$

received

April 28, 2017

accepted

February 8, 2018

published online

April 10, 2018
La localización más frecuente es la diáfisis o región diafiso-metafisaria de los huesos largos, siendo el fémur proximal (cuello y región intertrocanterea) y la tibia distal los huesos más afectados. ${ }^{2}$

El síntoma principal es el dolor, por lo general nocturno, que se alivia con la administración de antiinflamatorios no esteroideos y salicilatos. Otros signos o síntomas son edema local y dolor a la palpación, principalmente cuando su localización es subcutánea. Si está cercano a una articulación o es intra-articular puede tener derrame, sinovitis y limitación del rango articular.

El diagnóstico se basa en la sospecha clínica, apoyado por pruebas complementarias que confirmen la existencia del nidus. La tomografía computarizada (TC) suele ser la prueba

Copyright $\odot 2018$ Thieme Revinter

Publicações Ltda, Rio de Janeiro, Brazil
License terms

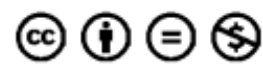


de imagen diagnóstica al permitir visualizar claramente el nidus como una pequeña lesión radiolúcida en el seno de la intensa reacción esclerótica, en ocasiones con una calcificación central. $^{3}$

El diagnóstico diferencial debe plantearse con el osteoblastoma, donde el tamaño del nidus es mayor $(>1,5 \mathrm{~cm})$, fracturas de estrés ${ }^{4}$ y con el raro osteosarcoma intracortical. En las presentaciones intraesponjosas también hay que considerar el absceso de Brodie. ${ }^{5}$

El tratamiento no quirúrgico con salicilatos o antiinflamatorios no esteroideos puede estar indicado, ya que esos medicamentos alivian el dolor. ${ }^{6}$ Sin embargo, existen pocos estudios que evalúen los resultados prolongados y que hayan publicado la resolución completa de los síntomas al retirar la medicación. En los casos donde el dolor es severo y no responden a la medicación el tratamiento más indicado actualmente es la radioablación percutánea guiada por TC. ${ }^{7,8}$

\section{Caso Clínico}

Presentamos el caso de una mujer de 27 años, diestra, estudiante, sin antecedentes personales de interés, que acude a la consulta de Traumatología de nuestro Hospital por dolor en la muñeca izquierda de un año de evolución. Refiere antecedente traumático con la muñeca en extensión meses antes de la consulta. No practica deporte de forma habitual.

El dolor según relata, es progresivo, aumenta al cargar peso y en los últimos meses le despierta por la noche y parece ceder con antiinflamatorios.

Inicialmente se le realiza estudio de radiografía simple para descartar fractura aguda o de estrés, dado el componente traumático (-Fig. 1A-B). Ante la ausencia de lesiones óseas y la persistencia del dolor tras varios meses, se realiza Resonancia magnética (RM) en busca de lesiones de partes blandas. Se diagnostica de edema óseo y tendinitis de segundo compartimento extensor (-Fig. 2A-C).
La persistencia de la sintomatología nos hace sospechar el OO como posible diagnóstico. Se solicita TC que objetiva una lesión osteolítica de margen escleroso, de $6 \mathrm{~mm}$ de diámetro y localización epifisaria central en el extremo distal del radio. Coincidiendo con dicha lesión se encuentra esclerosis en la médula ósea contigua a la lesión y derrame articular en articulación radioescafoidea (-Fig. 3A, B, C).

Se realiza una gammagrafía con Tc 99 que confirma el diagnostico de $\mathrm{OO}$ en estadio activo (-Fig. 4A-C).

La localización de la lesión a una distancia suficiente de la cortical ósea, articulación y partes blandas hace posible proponer la ablación por radiofrecuencia como tratamiento. Se le explica el procedimiento a la paciente así como la posibilidad de que éste no diese el resultado previsto, y fuese necesaria la extirpación quirúrgica.

Previo al tratamiento se realiza una medición de la fuerza de presa mediante dinamómetro Jamar ${ }^{\circledR}$ y cuestionario DASH para así poder comparar resultados a los 6 meses de evolución. Los registros de la fuerza de presa y cuestionario DASH previos al tratamiento fueron $5 \mathrm{~kg}$. y 31 puntos.

La paciente ingresa el mismo día de la intervención, que se realiza en la sala del escáner por un equipo multidisciplinar que incluye anestesista, radiólogo, técnico de rayos y traumatólogo.

Bajo sedación de la paciente, monitorización y tratamiento del dolor, se localiza la lesión mediante TC. Posteriormente, a través de una pequeña incisión se introduce una aguja de biopsia ósea que atraviesa la cortical y se toma muestra para anatomía patológica. A continuación se retira el fiador de la aguja de biopsia y se introduce el electrodo de punta fría. Una vez confirmada la localización correcta del electrodo se conecta al generador de radiofrecuencia y se mantiene a una temperatura de $90^{\circ}$ durante 6 minutos (-Fig. 5A-C).

El sistema utilizado en nuestro caso es el generador de radiofrecuencia Cool-tip E series, con un electrodo con $1 \mathrm{~cm}$. $(0,7 \mathrm{~cm}$ de punta activa).
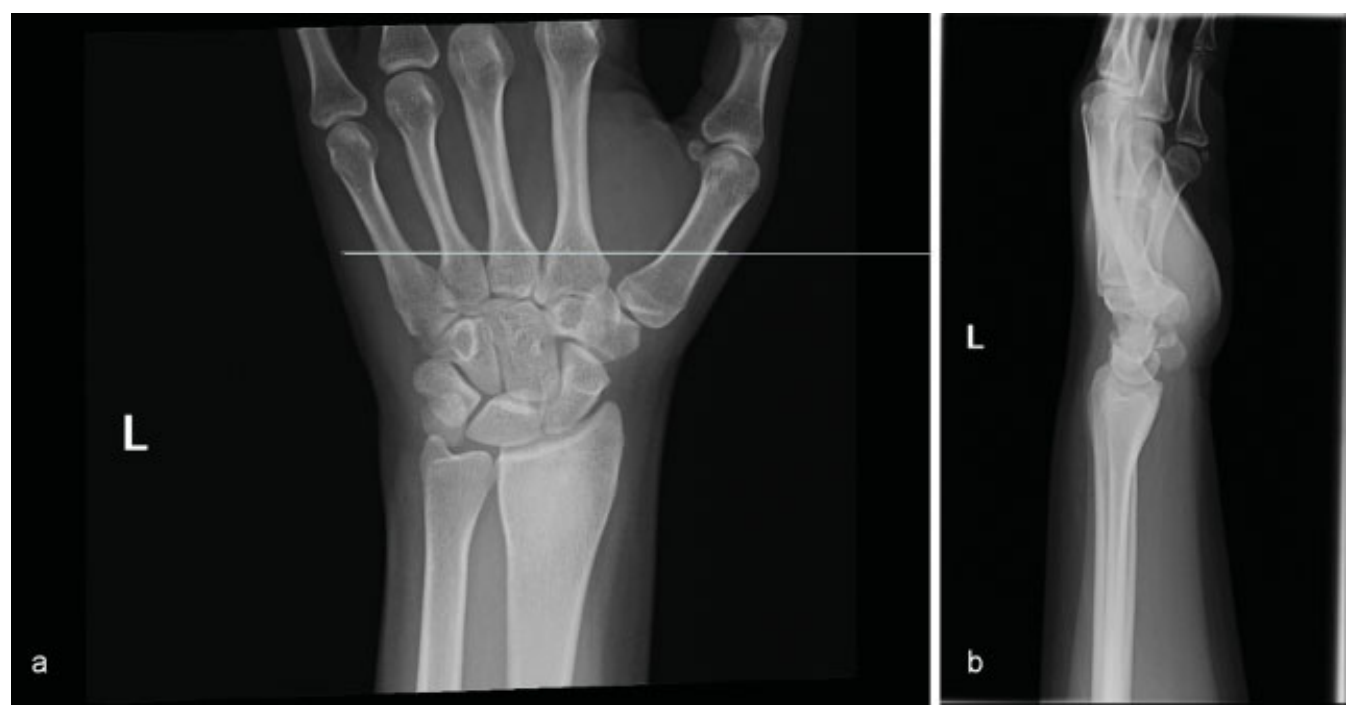

Fig. 1 (A) radiografía inicial AP. (B) radiografía inicial lateral. 


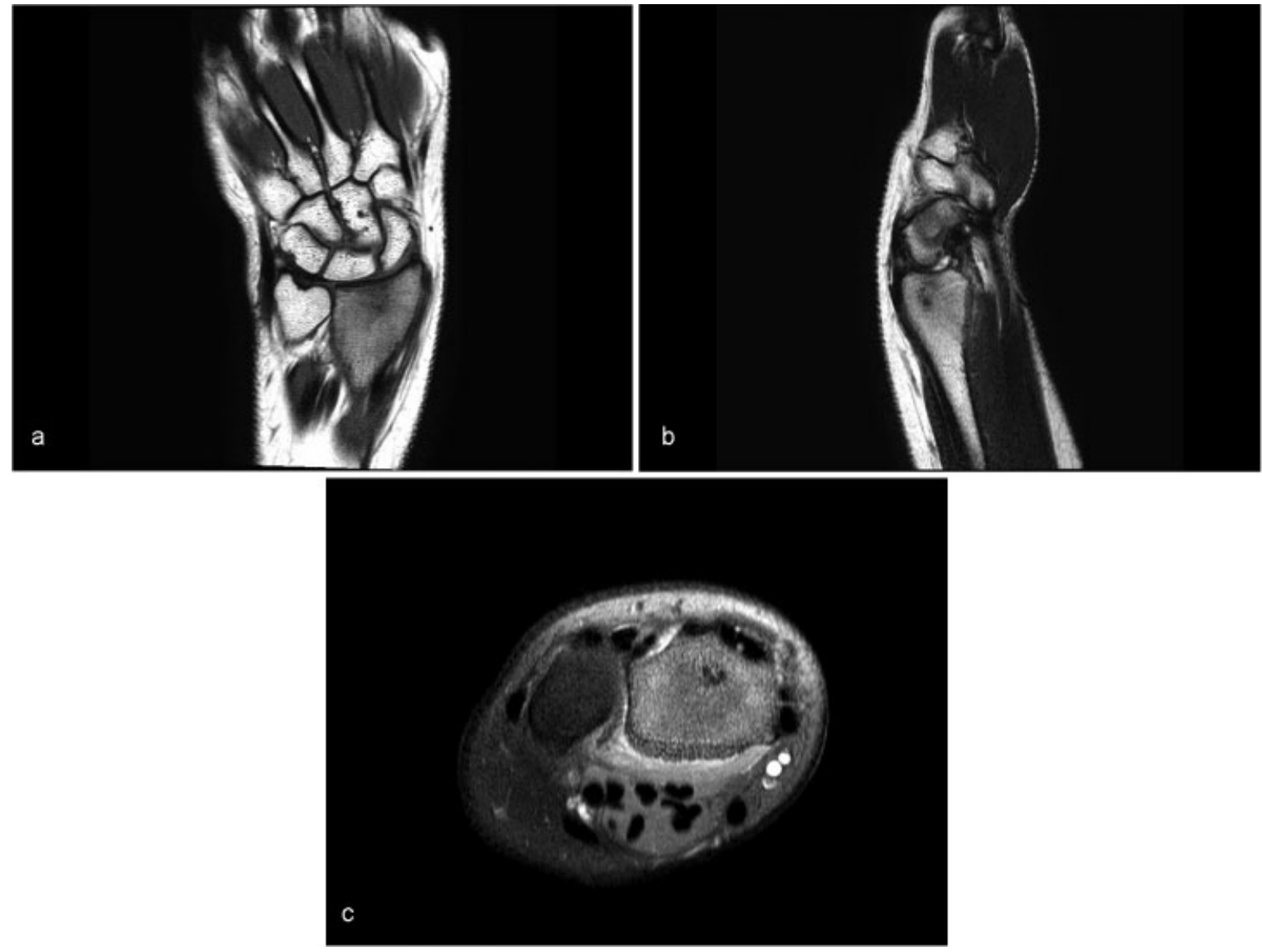

Fig. 2 (A) RMN, AP. (B) RMN, sagital. (C) RMN axial.
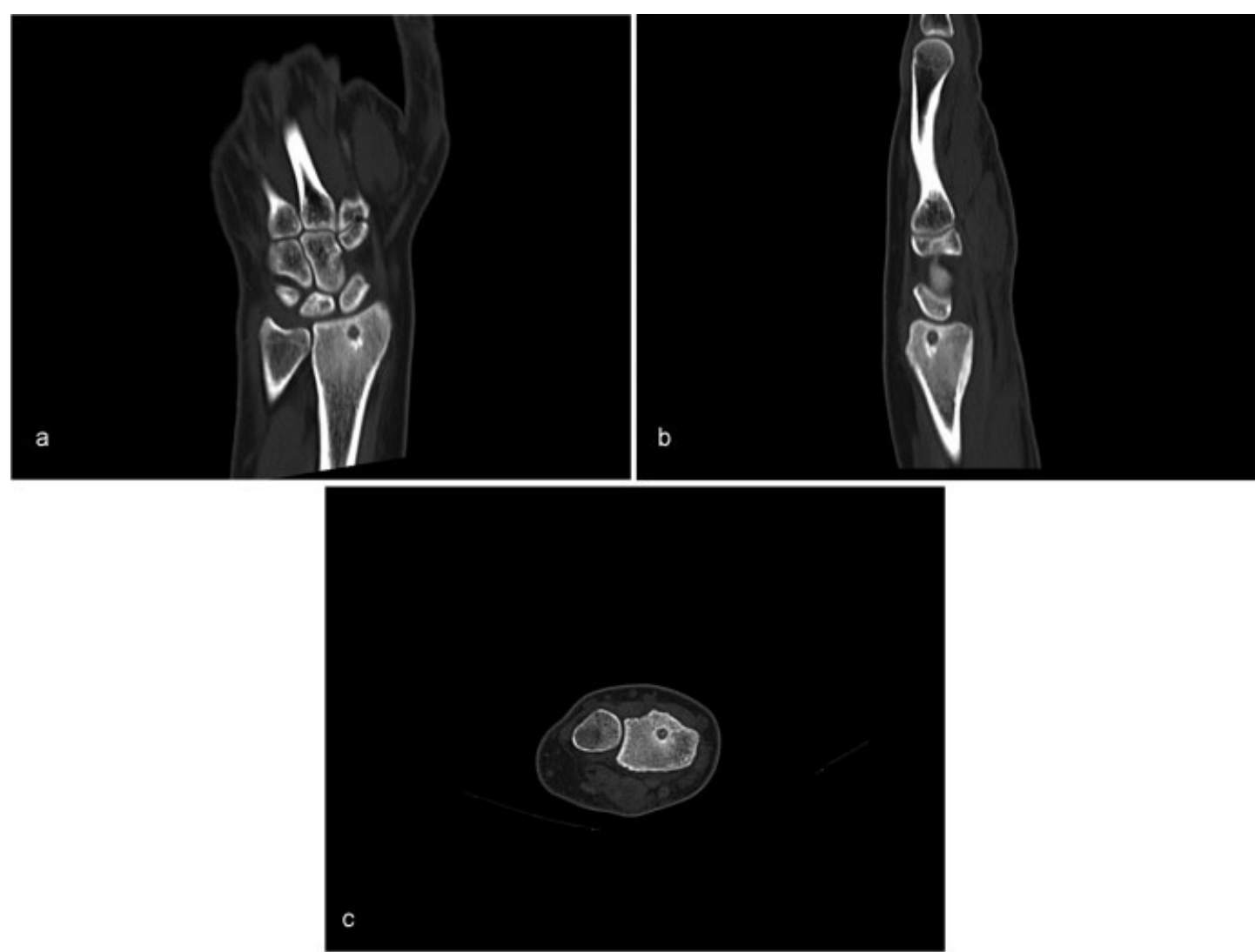

Fig. 3 (A) TC, corte coronal. Imagen compatible con nidus. (B) TC, corte sagital. (C) TC, corte axial. 

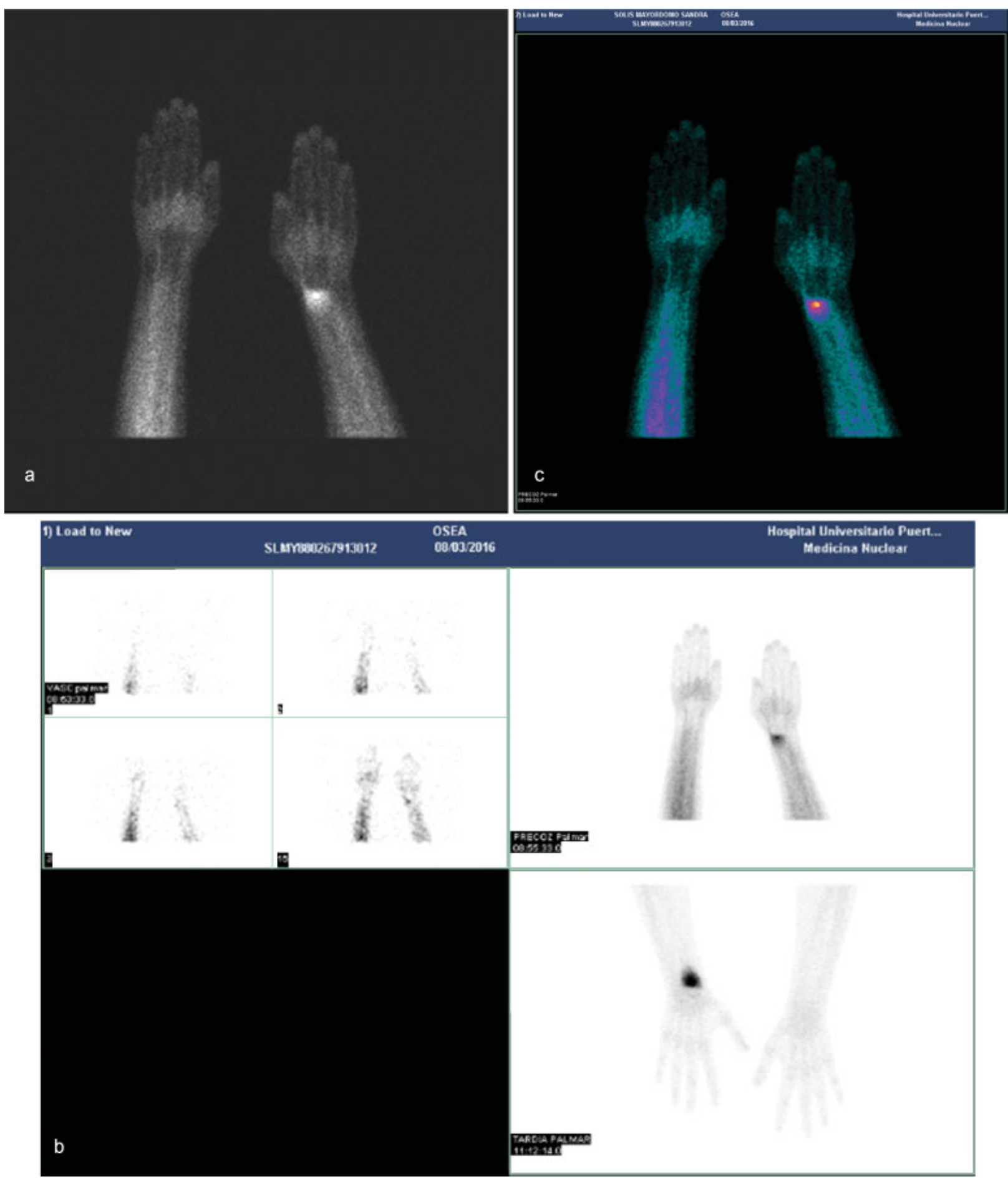

Fig. 4 (A-C) gammagrafia, con captación en lesión.

La paciente permanece ingresada por 24 horas en la planta de Traumatología para control de dolor tras la intervención.

La mejoría clínica de la paciente es inmediata. A las 6 semanas, acude a consulta asintomática, refiere iniciar capacidad para levantar pesos y hacer presa sin limitaciones.

La fuerza de prensión medida con dinamómetro de Jamar ${ }^{\circledR}$, a los 3 meses es de $10 \mathrm{~kg}$. y a los 6 meses de $14 \mathrm{~kg}$. (5 kg. previo al tratamiento). En el cuestionario DASH a los 6 meses de la intervención obtuvo 13 puntos (31 previo a tratamiento).

\section{Discusión}

El OO fue reconocido como entidad clínica independiente por Jaffe en $1935 .^{9}$ Se localiza preferentemente en las extremidades. Es raro en el esqueleto axial salvo en la columna. La extremidad inferior se ve afectada con mayor frecuencia que la superior. El fémur y la tibia suponen el $50 \%-$ $60 \%$ de los casos seguidos del pie, donde tiene predilección por el cuello del astrágalo. 

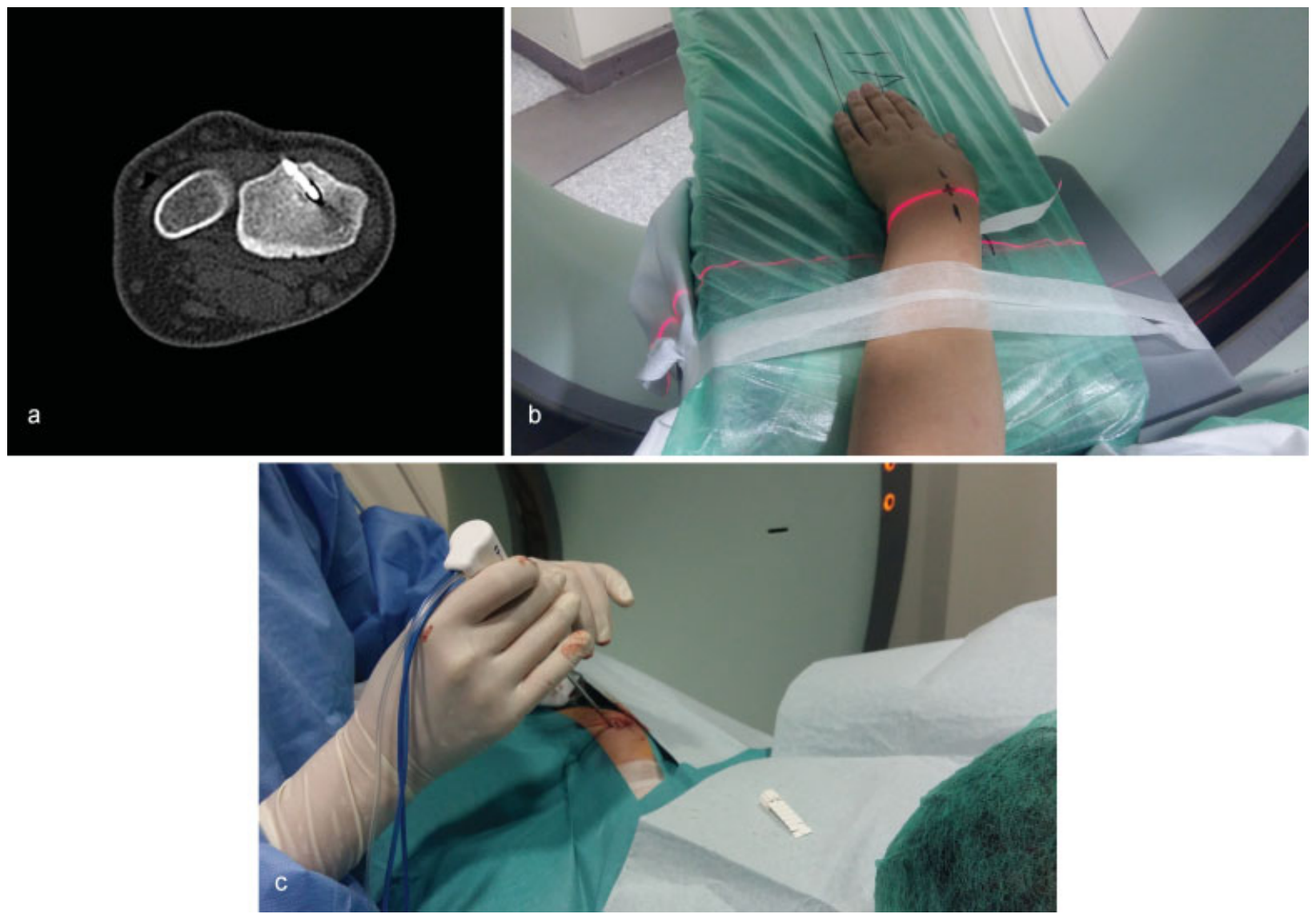

Fig. 5 (A) imagen de TC con punta de electrodo en lesión. (B) localización de la lesión guiada por TC. (C) imagen de intervención.

En cuanto a la extremidad superior, la mano es la localización más frecuente del $\mathrm{OO}$, siendo las falanges su principal asiento. ${ }^{9}$

El hueso cortical se afecta más que el hueso esponjoso. La localización metafisaria o diafisaria es lo más frecuente siendo más rara la afectación epifisaria.

La fisiopatología del 00 es controvertida. La naturaleza tumoral de la lesión ha sido fuente de debate, parece que las anormalidades encontradas en estudios citogenéticos en el cromosoma 22q avalan su origen neoplásico Otras teorías responsabilizan a los microtraumatismos y las fracturas de estrés como causas de su aparición.

El OO se considera un tumor activo (Estadio 2 de Enneking). Rara vez aumentan de tamaño y por lo general su dimensión es menor de $1 \mathrm{~cm}$. Puede volverse asintomático y curar espontáneamente, casos en los que los síntomas persisten de 3 a 5 años y poco a poco ceden con osificación de su nidus, pero el hueso reactivo persiste durante años. No se han observado casos de malignización.

El tratamiento de elección es la exéresis completa del nidus a excepción de aquellas lesiones que sean poco dolorosas, o que estén localizadas en zonas de difícil abordaje quirúrgico. En ocasiones el cuadro de dolor desaparece al cabo de varios años en pacientes que toman medicación de forma ocasional. ${ }^{10}$

La resección quirúrgica en bloque puede plantear problemas motivados por la dificultad de encontrar la lesión, lo que lleva a resecar grandes cantidades de hueso que supone el debilitamiento óseo y aumenta el riesgo de fractura.
Otro procedimiento quirúrgico descrito que trata de evitar las posibles complicaciones de la resección en bloque y con el que se han obtenido buenos resultados, consiste en realizar una exéresis de forma gradual del hueso reactivo, y una vez localizado el nidus legrarlo con cucharillas o con fresas de alta velocidad.

Con el fin de disminuir la agresión quirúrgica, se han propuesto otras técnicas para localizar y extirpar el nidus como la localización por TC y resección con trefina, la alcoholización, la fotocoagulación con láser o la termocoagulación con radiofrecuencia propuesta por Rosenthal. ${ }^{11,12}$ Esa última es la técnica que realizamos a los pacientes con osteoma osteoide en nuestro centro.

Aunque la mayoría de los $\mathrm{OO}$ son de diagnóstico sencillo, siguen presentándose algunos casos, como el paciente expuesto, cuyas localizaciones peculiares o tamaño pequeño pueden producir errores o retrasos en el diagnóstico.

La radiología simple en ocasiones no es significativa; en cambio, la TC muestra unos hallazgos patognomónicos de $\mathrm{OO}$ con las apariencias clásicas de un nidus de baja atenuación con calcificación central focal y esclerosis perinidal. La RM muestra edema dentro de la médula ósea, la cual siempre se limita al hueso afectado. ${ }^{2,13}$

El diagnóstico es clínico y radiológico. No es preciso un estudio anatomopatológico, puesto que la confirmación histológica ocurre en el $18-41 \%$ de los casos.

Para su tratamiento, se prefiere el tratamiento localizado de la lesión mediante radioablación, reservándose la cirugía 
abierta para aquellos casos en los que el diagnostico sea dudoso o el tumor esté localizado próximo a estructuras neurovasculares. Es imprescindible la colaboración estrecha y sincronizada de un equipo multidisciplinar compuesto por traumatólogo, radiólogo, técnico de rayos y anestesista.

\section{Conflicto de Intereses}

Los autores declaran que no hay conflicto de intereses.

\section{Bibliografía}

1 Frassica FJ, Waltrip RL, Sponseller PD, Ma LD, McCarthy EF Jr. Clinicopathologic features and treatment of osteoid osteoma and osteoblastoma in children and adolescents. Orthop Clin North Am 1996;27(03):559-574

2 Moberg E. The natural course of osteoid osteoma.J Bone Joint Surg Am 1951;33 A(01):166-170

3 Herrlin K, Ekelund L, Lövdahl R, Persson B. Computed tomography in suspected osteoid osteomas of tubular bones. Skeletal Radiol 1982;9(02):92-97

4 Giombini A, Dragoni S, Averna T, Ripani M, Longo UG, Maffulli N. Osteoid osteoma mimicking overuse syndromes in athletes. J Sports Med Phys Fitness 2009;49(02):167-170
5 Sim FH, Dahlin CD, Beabout JW. Osteoid-osteoma: diagnostic problems. J Bone Joint Surg Am 1975;57(02):154-159

6 Kneisl JS, Simon MA. Medical management compared with operative treatment for osteoid-osteoma. J Bone Joint Surg Am 1992;74(02):179-185

7 Kitsoulis P, Mantellos G, Vlychou M. Osteoid osteoma. Acta Orthop Belg 2006;72(02):119-125

8 Bisbinas I, Georgiannos D, Karanasos T. Wide surgical excision for osteoid osteoma. Should it be the first-choice treatment? Eur J Orthop Surg Traumatol 2004;14(03):151-154

9 Jaffe HL. Osteoid-osteoma. A benign osteoblastic tumor composed of osteoid and atypical bone. Arch Surg 1935;31:709-728

10 Martel Villagrán J, Bueno Horcajadas A, Ortiz Cruz EJ. [Percutaneous radiofrequency ablation of benign bone tumors: osteoid osteoma, osteoblastoma, and chondroblastoma]. Radiologia 2009;51(06): 549-558

11 Rosenthal DI, Alexander A, Rosenberg AE, Springfield D. Ablation of osteoid osteomas with a percutaneously placed electrode: a new procedure. Radiology 1992;183(01):29-33

12 Rosenthal DI, Springfield DS, Gebhardt MC, Rosenberg AE, Mankin HJ. Osteoid osteoma: percutaneous radio-frequency ablation. Radiology 1995;197(02):451-454

13 McGrath BE, Bush CH, Nelson TE, Scarborough MT. Evaluation of suspected osteoid osteoma. Clin Orthop Relat Res 1996;(327): 247-252 